\title{
Characterization of some Classes Related to the Class of Browder Linear Relations
}

\author{
Attaif Farah, Maher Mnif \\ Department of Mathematics, Faculty of Science of Sfax, University of Sfax \\ Soukra Road, Km 3.5, PO Box 1171, 3000, Sfax, Tunisia \\ farah-lotfi1@hotmail.fr, maher.mnif@gmail.com
}

Abstract: In this paper, we introduce the sets of left and right invertible linear relations and we give some of their properties. Furthermore, we study the connection between these sets and the classes of Fredholm linear relations. The obtained results are used to give some characterizations of some classes related to the class of Browder linear relations.

Key words: Closed linear relation, left- and right-Browder linear relation, left and right invertible linear relation.

AMS Subject Class. (2000): Primary 47A06, 47A53, 47A05.

\section{INTRODUCTION}

First, let us notice that, throughout this paper, $(X,\|\|)$ and $(Y,\|\|)$ will represent complex Banach spaces. A linear relation $T: X \rightarrow Y$ is a mapping from a subspace $D(T)=\{u \in X: T(u) \neq \emptyset\} \subseteq X$, called the domain of $T$, which takes values in $\mathcal{P}(Y) \backslash\{\emptyset\}$ (the collection of nonempty subsets of $Y$ ) and is such that $T\left(\alpha x_{1}+\beta x_{2}\right)=\alpha T\left(x_{1}\right)+\beta T\left(x_{2}\right)$ for all non-zero scalars $\alpha, \beta \in \mathbb{K}$ and $x_{1}, x_{2} \in D(T)$. The class of all linear relations from $X$ to $Y$ will be denoted by $\mathcal{L R}(X, Y)$. We write $\mathcal{L} \mathcal{R}(X)=\mathcal{L} \mathcal{R}(X, X)$.

If $T$ maps the points of its domain to singletons, then $T$ is said to be an operator, which is equivalent to $T(0)=\{0\}$. The class of linear bounded operators defined on all $X$ is denoted by $B(X, Y)$. A linear relation $T$ is uniquely defined by its graph $G(T)=\{(u, v) \in X \times Y: u \in D(T), v \in T(u)\}$.

The inverse of $T$ is the relation $T^{-1}$ given by:

$$
G\left(T^{-1}\right)=\{(v, u) \in Y \times X:(u, v) \in G(T)\} .
$$

If $G(T)$ is closed, then $T$ is said to be closed. The class of such relations is denoted by $C R(X, Y)$. We denote by $R(T)=T(D(T))$ the range of $T$ and 
by $N(T):=\{x \in X:(x, 0) \in G(T)\}$ the kernel of $T$. If $R(T)=Y$, then $T$ is called surjective, and if $N(T)=\{0\}$, then $T$ is called injective. We may write $n(T)=\operatorname{dim} N(T)$ and $d(T)=\operatorname{codim} R(T)$ and the index of $T$, namely $i(T)$, is defined by $i(T)=n(T)-d(T)$, provided that $n(T)$ and $d(T)$ are not both infinite.

For $S, T \in \mathcal{L R}(X, Y)$ and $\lambda \in \mathbb{K}$, the linear relations $S+T, S \hat{+} T, S \oplus T$ and $\lambda S$ are defined by $G(S+T):=\{(x, y+z) \in X \times Y:(x, y) \in G(S)$ and $(x, z) \in$ $G(T)\}, G(S \hat{+} T):=\{(x+y, z+t) \in X \times Y:(x, z) \in G(S)$ and $(y, t) \in G(T)\}$, this last sum is direct when $G(S) \cap G(T)=\{(0,0)\}$. In such case, we write $S \oplus T, G(\lambda S):=\{(x, \lambda y) \in X \times Y:(x, y) \in G(S)\}$, and $S \subset T$ means that $G(S) \subset G(T)$. For $T \in \mathcal{L} \mathcal{R}(X, Y)$ and $S \in \mathcal{L} \mathcal{R}(Y, Z)$, the product $S T$ is given by $G(S T):=\{(x, z) \in X \times Z:(x, y) \in G(T),(y, z) \in G(S)$ for some $y \in Y\}$.

Let $T \in \mathcal{L R}(X)$. If $\alpha \in \mathbb{K}$, then $\alpha-T$ stands for $\alpha I-T$, where $I$ is the identity operator in $X$. The resolvent set of $T$ is the set $\rho(T)=\{z \in \mathbb{C}$ : $\left.(z-T)^{-1} \in B(X)\right\}$. If $M$ is a subspace of $X$, then $T_{M}$ is the linear relation whose graph is $G(T) \cap(M \times M)$.

Recall that the class of upper semi-Fredholm linear relations is denoted by:

$$
\phi_{+}(X, Y)=\{T \in C R(X, Y): R(T) \text { is closed and } n(T)<\infty\} .
$$

Moreover, the class of lower semi-Fredholm linear relations is denoted by:

$$
\phi_{-}(X, Y)=\{T \in C R(X, Y): R(T) \text { is closed and } d(T)<\infty\} .
$$

$T$ is called a Fredholm relation, if $T \in \phi_{+}(X, Y) \cap \phi_{-}(X, Y)$. The class of all Fredholm relations is denoted by $\phi(X, Y)$.

Recall that a closed subspace $M$ in a normed space $X$ is said to be complemented in $X$ if there exists a closed subspace $N$ of $X$ such that $X=M+N$ and $\{0\}=M \cap N$ (in short, $X=M \oplus N)$ ).

If a linear relation $T \in \mathcal{L R}(X, Y)$ is upper semi-Fredholm and $R(T)$ is complemented in $Y$, then $T$ is said to be left-Fredholm linear relation. A linear relation $T \in \mathcal{L} \mathcal{R}(X, Y)$ is right-Fredholm relation if it is lower semi-Fredholm and $N(T)$ is complemented in $D(T)$. The set of left-Fredholm linear relations (right-Fredholm linear relations) is denoted by $\phi_{l}(X, Y)\left(\phi_{r}(X, Y)\right)$.

Let $T \in \mathcal{L} \mathcal{R}(X)$. We define $T^{n} \in \mathcal{L} \mathcal{R}(X), n \in \mathbb{N}$ by $T^{0}=I, T^{1}=T$ and $T^{n}=T T^{n-1}$. We define $N^{\infty}(T)=\cup_{n} N\left(T^{n}\right)$ and $R^{\infty}(T)=\cap_{n} R\left(T^{n}\right)$. The singular chain manifold of $T \in \mathcal{L R}(X), R_{c}(T)$ is defined by

$$
R_{c}(T):=\left(\bigcup_{n=1}^{\infty} N(T)\right) \bigcap\left(\bigcup_{n=1}^{\infty} T^{n}(0)\right) .
$$


The ascent and the descent of $T \in \mathcal{L} \mathcal{R}(X)$ are defined as follows:

$$
\begin{aligned}
\operatorname{asc}(T) & :=\min \left\{p \in \mathbb{N}: N\left(T^{p}\right)=N\left(T^{p+1}\right)\right\}, \\
\operatorname{des}(T) & :=\min \left\{p \in \mathbb{N}: R\left(T^{p}\right)=R\left(T^{p+1}\right)\right\},
\end{aligned}
$$

respectively, whenever these minima exists. If no such numbers exist, the ascent and descent of $T$ are defined to be $\infty$. A relation $T \in C R(X)$ is upper semi-Browder if it is upper semi-Fredholm with finite ascent. If $T \in$ $C R(X)$ is lower semi-Fredholm with finite descent, then $T$ is lower semiBrowder. Let $B_{+}(X)\left(B_{-}(X)\right)$ denotes the set of all upper (lower) semiBrowder linear relations. The set of Browder linear relations is defined by $B(X)=B_{+}(X) \cap B_{-}(X) . T \in C R(X)$ is said to be left-Browder relation if it is left-Fredholm with finite ascent. If $T \in C R(X)$ is right-Fredholm with finite descent, then $T$ is right-Browder relation. Let $B_{l}(X)\left(B_{r}(X)\right)$ denotes the set of all left-(right-) Browder linear relations.

A study of left and right Browder linear relations has been carried by a number of authors in the recent past (see [5], [7], [9]). In a recent paper of (2016) [7], the authors prove that a left (right) Browder linear relation $T$ in a Banach space can be expressed in the form $T=A+B$ where $A$ is an injective (onto) left (right) Fredholm linear relation and $\mathrm{B}$ is a bounded finite rank operator with $B T \subset T B$.

The purpose of the present paper is to consider the notion of left and right invertible linear relations and we give some characterizations of left- and right-Browder closed linear relations.

To make the paper easily accessible, some results from the theory of linear relations due to Cross [8] are recalled in Section 2. In Section 3, we extend to the general case of closed linear relations in Banach spaces, some results concerning upper and the lower semi-Browder closed operators proved by Sne žana $\breve{C}$ in [11, Theorem 3 and Theorem 4]. In particular, we prove that the upper (lower) semi-Browder linear relation $T$ is a upper (lower) semi-Fredholm and almost bounded below (onto) linear relation. Finally, in Section 4, the definition of left (right) invertible linear relation is given, and some properties of these relations are shown wich have been used to characterize the left (right) Browder linear relations. In particular, we prove that the linear relation $T$ is left (right) Browder if and only if there exists a bounded operator projector $P$, such that $T P-P T=T-T, \operatorname{dim} R(P)<\infty,(T P)^{d}=T(0)$ for some $d \in \mathbb{N}$ and $T+P$ is left (right) invertible linear relation if and only if there exists a compact operator $B$ satisfying $T B-B T=T-T$ and $T-B$ is a left (right) invertible linear relation. These results are generalizations of the results in the 
case of linear operators shown by Sne ̌aana $\breve{C}$. Z̆ivković-Zlatanović, Dragan S. Djordjević and Robin E. Harte [11, Theorem 5 and Theorem 6].

\section{Auxiliary Results}

In this section, we recall some auxiliary results from the theory of linear relations in Banach spaces.

Let $T$ be a linear relation in a Banach space $X$. Recall that $T$ is said to be continuous if for each neighborhood $V$ in $R(T)$, the inverse image $T^{-1}(V)$ is a neighborhood in $D(T)$, bounded if it is continuous and its domain is whole $X$, open if its inverse is continuous.

In order to give some characterizations of these classes of linear relations, one introduces the following notations. Let $Q_{T}$ denotes the quotient map from $X$ onto $X / T(0)$. We note that $Q_{T} T$ is single-valued and so we can define $\|T x\|:=\left\|Q_{T} T x\right\|, x \in D(T)$ and $\|T\|:=\left\|Q_{T} T\right\|$ called the norm of $T x$ and $T$ respectively, and the minimum modulus of $T$ is the quantity

$$
\gamma(T):=\sup \{\lambda \geq 0: \lambda \operatorname{dist}(x, N(T)) \leq\|T x\|, x \in D(T)\} .
$$

In [8, II.3.2 and II.5.3] Ronald Cross proves that:

(i) $T$ is continuous if and only if $\|T\|<\infty$;

(ii) $T$ is open if and only if $\gamma(T)>0$;

(iii) $T$ is closed if and only if $Q_{T} T$ is a closed operator and $T(0)$ is a closed subspace.

Recall that $T$ is said to be regular linear relation if $R(T)$ is closed and $T$ verifies one of the equivalent conditions:

(i) $N(T) \subseteq R\left(T^{m}\right)$, for all nonnegative integer $m$;

(ii) $N\left(T^{n}\right) \subseteq R(T)$, for all nonnegative integer $n$;

(iii) $N\left(T^{n}\right) \subseteq R\left(T^{m}\right)$, for all nonnegative integers $n$ and $m$.

The Kato decomposition of left and right-Fredholm linear relations are collected in the following lemma.

Lemma 2.1. ([7, Theorem 5.1 and Theorem 6.1]) Let $T \in C R(X, Y)$. Then: 
(i) If $T \in \phi_{l}(X, Y)$, then there exist two closed subspaces $M$ and $N$ of $X$ such that $X=M \oplus N$ with $N \subset D(T)$ and $\operatorname{dim} N<\infty ; T=T_{M} \oplus T_{N}$, such that $T_{M}$ is a regular left-Fredholm linear relation in $M$ and $T_{N}$ is a bounded nilpotent operator in $N$.

(ii) If $T \in \phi_{r}(X, Y)$, be such that $\overline{D(T)}=X$ and $\rho(T) \neq \emptyset$, then there exist two closed subspaces $M$ and $N$ of $X$ such that $X=M \oplus N$ with $N \subset D(T)$ and $\operatorname{dim} N<\infty ; T=T_{M} \oplus T_{N}$, such that $T_{M}$ is a regular right-Fredholm linear relation in $M$ and $T_{N}$ is a bounded nilpotent operator in $N$.

Let $M, L$ be two subspaces of a Banach space $X$ and let

$$
\delta(M, L)=\sup _{x \in M\|x\| \leq 1} \operatorname{dis}(x, L) .
$$

The gap between $M$ and $L$ is defined by

$$
\widehat{\delta}(M, L)=\max \{\delta(M, L), \delta(L, M)\} .
$$

Finally, we give the main result of this section.

Theorem 2.1. Let $T$ be a bounded regular linear relation with $\rho(T) \neq \emptyset$. Then

(i) If $T$ is almost bounded below, then $T$ is bounded below.

(ii) If $T$ is almost onto, then $T$ is onto.

Proof. (i) We have $T$ is almost bounded below, then there exists $\delta>0$ such that $T-\lambda$ is injective and open for all $0<|\lambda|<\delta$. Hence $N(T-\lambda)=\{0\}$ and $\gamma(T-\lambda)>0$. On the other hand, we have $T$ is regular, then $\gamma(T)>0$. By using [2, Lemma 2.10 (ii)] and [1, Theorem 23(5)], we deduce that

$$
\begin{aligned}
\widehat{\delta}(N(T-\lambda), N(T)) & \leq \frac{|\lambda|}{\min \{\gamma(T-\lambda), \gamma(T)\}} \\
& \leq \frac{|\lambda|}{\gamma(T)-3|\lambda|} .
\end{aligned}
$$

Hence $\lim _{\lambda \rightarrow 0} \widehat{\delta}(N(T-\lambda), N(T))=0$. Therefore there exists $\lambda>0$ such that $\widehat{\delta}(N(T-\lambda), N(T))<1$. Thus by [10, Corollary 10] we have $\operatorname{dim} N(T)=$ $\operatorname{dim} N(T-\lambda)$. Then $N(T)=\{0\}$. Therefore $T$ is bounded below. 
(ii) Suppose that $T$ is almost onto, then there exists $\delta>0$ such that for all $0<|\lambda|<\delta$ we have $R(T-\lambda I)=X$. Hence $N\left((T-\lambda I)^{\prime}\right)=R(T-\lambda I)^{\perp}=\{0\}$. By [12, Proposition III.1.5] we have $N\left(T^{\prime}-\lambda I\right)=\{0\}$. Therefore $T^{\prime}$ is almost bounded below and regular relation. Then, by (i), we have $T^{\prime}$ is bounded below. Hence $N\left(T^{\prime}\right)=\{0\}$, then $R(T)=X$. Thus, $T$ is onto.

\section{Some Properties of UPPER AND LOWER SEMI-Browder LINEAR RELATIONS}

The goal of this section is to discuss some properties of upper and lower semi-Browder linear relations that will be used in the last section.

Definition 3.1. Let $T \in \mathcal{L R}(X)$ and $S \in B(X)$. We say that $S$ commutes with $T$ if $S(D(T))=D(T)$ and for all $x \in D(T)$, we have, STx $=T S x$. We shall write

$\operatorname{comm}_{\epsilon}^{-1}(T)=\{S \in B(X): S$ invertible, commutes with $T$ and $\|S\|<\epsilon\}$.

Proposition 3.1. Let $X$ be a Banach space and $T \in C R(X)$ be such that $\overline{D(T)}=X$ and $\rho(T) \neq \emptyset$. Let $S \in \operatorname{comm}_{\epsilon}^{-1}(T)$. Then

(i) $S^{-1}$ commutes with $T$.

(ii) $S^{\prime}$ commutes with $T^{\prime}$.

(iii) For all $n \in \mathbb{N}^{*}, S^{n}$ commutes with $T^{n}$.

Proof. (i) Let $x \in D(T)$. Then there exists $u \in D(T)$ such that $S u=x$. We have $T S u=S T u$, hence $T x=S T S^{-1} x$, and $S^{-1} T x=T S^{-1} x$. Therefore $S^{-1}$ commutes with $T$.

(ii) First we claim that $S^{\prime}\left(D\left(T^{\prime}\right)\right)=D\left(T^{\prime}\right)$. Indeed, for $y^{\prime} \in D\left(T^{\prime}\right)$ we have

$$
\left\|S^{\prime} y^{\prime}(T x)\right\|=\left\|\left(y^{\prime} S\right)(T x)\right\|=\left\|\left(y^{\prime} T\right)(S x)\right\| \leq\left\|y^{\prime} T\right\|\|S\|\|x\|
$$

for every $x \in D(T)$. Hence, $S^{\prime} y^{\prime} T$ is continuous. We have $S^{\prime} y^{\prime} T(0)=$ $S^{\prime}\left(T^{\prime} y^{\prime}(0)\right)=S^{\prime}(0)=0$, then, by [8, Proposition III.1.2] we deduce that $S^{\prime} y^{\prime} \in D\left(T^{\prime}\right)$. Therefore, $S^{\prime}\left(D\left(T^{\prime}\right)\right) \subset D\left(T^{\prime}\right)$. We have $S \in \operatorname{comm}_{\epsilon}^{-1}(T)$ then, $S^{\prime}$ is bijective and $\left\|S^{\prime}\right\|=\|S\|$. Let $y^{\prime} \in D\left(T^{\prime}\right)$. Then there exists a unique functional $z^{\prime} \in X^{\prime}$ such that $y^{\prime}=S^{\prime} z^{\prime}=z^{\prime} S$. It follows that $z^{\prime}=y^{\prime} S^{-1}$ and by (i) we get:

$$
\begin{aligned}
\left\|\left(z^{\prime} T\right) x\right\| & =\left\|y^{\prime}\left(S^{-1}(T x)\right)\right\|=\left\|y^{\prime}\left(T\left(S^{-1} x\right)\right)\right\| \\
& =\left\|\left(y^{\prime} T\right)\left(S^{-1} x\right)\right\| \leq\left\|y^{\prime} T\right\|\left\|S^{-1}\right\|\|x\| .
\end{aligned}
$$


Therefore, $z^{\prime} T$ is continuous. We have $z^{\prime} T(0)=y^{\prime} S^{-1} T(0)=y^{\prime} T(0)=0$, then, by [8, Proposition III.1.2] we deduce that $z^{\prime} \in D\left(T^{\prime}\right)$. Now, we show that $T^{\prime} S^{\prime}=S^{\prime} T^{\prime}$. We have $S$ and $S^{\prime}$ are bijective, then $R\left(S^{\prime}\right)=X^{\prime}, D\left(S^{\prime}\right)=X^{\prime}$, $D(T) \subset R(S)=X$, and $R(T) \subset D(S)=X$. Hence by [9, Theorem III.1.6], we have $(S T)^{\prime}=T^{\prime} S^{\prime}$ and $(T S)^{\prime}=S^{\prime} T^{\prime}$. Therefore $T^{\prime} S^{\prime}=(S T)^{\prime}=(T S)^{\prime}=S^{\prime} T^{\prime}$. Then $S^{\prime}$ commutes with $T^{\prime}$.

(iii) For $n=2$ we will show that $S^{2}\left(D\left(T^{2}\right)\right)=D\left(T^{2}\right)$ and for all $x \in$ $D\left(T^{2}\right)$, we have, $S^{2} T^{2} x=T^{2} S^{2} x$. Indeed, let $x \in D\left(T^{2}\right)$. Then $x \in D(T)$ and $T x \cap D(T) \neq \emptyset$. Using that $x \in D(T)$ and $S(D(T))=D(T)$, we get $S^{2} x \in D(T)$. On another hand, we have $T S^{2} x=S^{2} T x, T x \cap D(T) \neq \emptyset$ and $S(D(T))=D(T)$ then $T S^{2} x \cap D(T) \neq \emptyset$. So, $S^{2} x \in D\left(T^{2}\right)$. Therefore $D\left(T^{2}\right) \subset S^{2}\left(D\left(T^{2}\right)\right)$. Let $x \in D\left(T^{2}\right)$. Then $x \in D(T)$ and $T x \cap D(T) \neq \emptyset$. We have $S(D(T))=D(T)$, then there exists $y \in D(T)$ such that $x=S^{2} y$. It remains to prove that $T y \cap D(T) \neq \emptyset$. We have,

$$
T x \cap D(T)=T S^{2} y \cap D(T)=S^{2} T y \cap D(T) \neq \emptyset .
$$

Then $T y \cap D(T) \neq \emptyset$. Hence $S^{2}\left(D\left(T^{2}\right)\right) \subset D\left(T^{2}\right)$. Therefore $S^{2}\left(D\left(T^{2}\right)\right)=$ $D\left(T^{2}\right)$. Let $x \in D\left(T^{2}\right)$. Then

$$
S^{2} T^{2} x=S S T T x=S T S T x=\operatorname{TSTS} x=\operatorname{TTSS} x=T^{2} S^{2} x .
$$

The case $n>2$, is deduced by using an induction argument.

Now, we prove the following result useful for the proof of the first main result of this section.

Proposition 3.2. Let $T \in \mathcal{L} \mathcal{R}(X)$ and $S \in \operatorname{comm}_{\epsilon}^{-1}(T)$. Then

$$
N(T-S) \subset R^{\infty}(T) .
$$

Proof. First we show by induction that, if $x \in N(T-S)$ then for all $n \geq 1$, we have $T^{n} x=S^{n} x+T^{n}(0)$. The case $n=1$ is obvious. Assume that $T^{n} x=S^{n} x+T^{n}(0)$ and we shall prove that $T^{n+1} x=S^{n+1} x+T^{n+1}(0)$. Indeed,

$$
\begin{aligned}
T^{n+1} x & =T S^{n} x+T T^{n}(0)=S^{n} T x+T^{n+1}(0) \\
& =S^{n}(S x+T(0))+T^{n+1}(0)=S^{n+1} x+T^{n+1}(0) .
\end{aligned}
$$

Now, let $x \in N(T-S)$. We have $S^{-1}$ commutes with $T$, then for all $n \geq 1$, $T^{n}\left(S^{-1}\right)^{n} x=\left(S^{-1}\right)^{n} T^{n} x=x+T^{n}(0)$. Hence $N(T-S) \subset R\left(T^{n}\right)$. Therefore $N(T-S) \subset R^{\infty}(T)$. 
Now, we are ready to state the first main result of this section.

Theorem 3.1. Let $T \in B_{+}(X)$ be such that $R_{c}(T)=\{0\}$. Then, the following statements holds:

(i) $T \in \phi_{+}(X)$, and there exists $\epsilon>0$ such that for all $S \in \operatorname{comm}_{\epsilon}^{-1}(T)$, we have $T-S$ is bounded below.

(ii) $T \in \phi_{+}(X)$, and almost bounded below.

Proof. (i) We have $T \in \phi_{+}(X)$, then, by [5, Lemma 2.5], $T^{n} \in \phi_{+}(X)$ for all $n \in \mathbb{N}$. Let $X_{1}=R^{\infty}(T)$. Then $X_{1}$ is a closed subspace. Let $T_{1}: X_{1} \rightarrow X_{1}$; the restriction of $T$ to $X_{1}$. Then, by using [1, Lemma 20] we deduce that $\beta\left(T_{1}\right)=0$ and $\alpha\left(T_{1}\right)<\infty$. Then, $T_{1} \in \phi(X)$.

Clearly we have $S\left(X_{1}\right) \subset X_{1}$. Writing $S_{1}: X_{1} \rightarrow X_{1}$, the restriction of $S$ to $X_{1}$. Then, by using [12, Proposition 2.4 and Proposition 2.6], we deduce that $\alpha\left(T_{1}-S_{1}\right) \leq \alpha\left(T_{1}\right) ; \beta\left(T_{1}-S_{1}\right) \leq \beta\left(T_{1}\right)$, and $i\left(T_{1}-S_{1}\right)=i\left(T_{1}\right)$.

From Proposition 3.2 we deduce that $\alpha(T-S)=\alpha\left(T_{1}-S_{1}\right)$. Therefore $\alpha(T-S)=i\left(T_{1}-S_{1}\right)=i\left(T_{1}\right)=\alpha\left(T_{1}\right)$. Now, by using $R_{c}(T)=\{0\}$ and $\operatorname{asc}(T) \leq p$ for some $p \in \mathbb{N}$, we get $N\left(T^{k}\right) \cap R\left(T^{p}\right)=\{0\}$ for all $k \in \mathbb{N}$. Hence $\alpha\left(T_{1}\right)=0$ and therefore $\alpha(T-S)=0$. Furthermore by [4, Proposition 3], $T-S$ has a closed range, then $T-S$ is bounded below.

(ii) is obvious.

Now, we are in position to give the second main result of this section.

Theorem 3.2. Let $X$ be a Banach space and $T \in B_{-}(X)$ be such that $\overline{D(T)}=X$ and $\rho(T) \neq \emptyset$. Then

(i) $T \in \phi_{-}(X)$ and there exists $\epsilon>0$ such that for all $S \in \operatorname{comm}_{\epsilon}^{-1}(T)$, we have $T-S$ is onto.

(ii) $T \in \phi_{-}(X)$ and almost onto.

Proof. (i) Let $T \in B_{-}(X)$ be such that $\overline{D(T)}=X$ and $\rho(T) \neq \emptyset$. Then, by using [6, Theorem 2.1], [5, Lemma 2.3] and [8, V.1.1] we deduce that $T^{\prime} \in B_{+}(X)$ and $R_{c}\left(T^{\prime}\right)=0$. Hence by Theorem 3.1, there exists $\epsilon>0$ such that for all $A \in \operatorname{comm}_{\epsilon}^{-1}\left(T^{\prime}\right), T^{\prime}-A$ is bounded below.

Let $S \in \operatorname{comm}_{\epsilon}^{-1}(T)$. Then by Proposition 3.1, we have $S^{\prime} \in \operatorname{comm}_{\epsilon}^{-1}\left(T^{\prime}\right)$. Therefore $\beta(T-S)=\alpha\left((T-S)^{\prime}\right)=\alpha\left(T^{\prime}-S^{\prime}\right)=0$. Then $T-S$ is onto.

(ii) is obvious. 


\section{Characterization of Left- And Right-Browder LINEAR RELATIONS}

This section concerns the characterization of left- and right-Browder linear relations in Banach spaces.

4.1. Characterization of left-Browder linear relations. We begin by introducing the new concept of left invertible linear relation and give some of its properties.

Definition 4.1. Let $T \in \mathcal{L} \mathcal{R}(X)$. We say that $T$ is left invertible, if there exists a bounded operator $A$ such that for all $x \in D(T), A T x=x$. In this case we say that $A$ is a left inverse of $T$.

The following lemmas give the relationship between the notion of bounded below linear relations and the notion of left invertible linear relations.

Lemma 4.1. Let $T$ be an everywhere defined closed bounded below linear relation. If $R(T)$ is complemented in $X$, then $T$ is left invertible.

Proof. Since $T$ is injective and open then $T^{-1}$ is a continuous operator. We have $R(T)$ is complemented, then there exists a closed subspace $F$ of $X$ such that $X=R(T) \oplus F$ and there exists a continuous projector $P$ such that $R(P)=F$ and $N(P)=R(T)$. Take $A=T^{-1}(I-P)+P$. Then $A$ is a bounded operator and we have for all $x \in D(T), A T x=x$. Therefore $T$ is left invertible.

Lemma 4.2. Let $T$ be a closed left invertible linear relation. Then $T$ is bounded below.

Proof. Let $A$ be a bounded operator such that for all $x \in D(T), A T x=x$. Let $x \in N(T)$. Then $T(x)=T(0)$. Hence $x=A T(x)=A T(0)=0$. Therefore $N(T)=\{0\}$.

On the other hand, for all $x \in D(T), T^{-1} T(x)=x=A T(x)$. Then for all $y \in R(T)$ we have $T^{-1}(y)=A(y)$. Hence $\left\|T^{-1} y\right\|=\|A y\| \leq\|A\|\|y\|$, for all $y \in D\left(T^{-1}\right)$. Hence $T^{-1}$ is a continuous relation. Thus $T$ is bounded below.

Proposition 4.1. Let $T$ be an everywhere defined closed left invertible linear relation on a Banach space $X$ such that $T(0)$ is complemented. Then $T \in \phi_{l}(X)$. 
Proof. Using Lemma 4.2 and [8, V.18] we deduce that $T \in \phi_{+}(X)$. Let $A$ be a left inverse of $T$, we have $(T A)^{2} x=T A T A x=T A x$. Then $T A$ is a multivalued projector. Let $N=R(T)=R(T A)=N(I-T A)$ and $M=N(T A)=R(I-T A)$. We claim that $M \cap N=T(0)$. Indeed, let $x \in M \cap N$. Then there exists $y \in X$ such that $x \in T A y$ and $0 \in T A x$. Hence $0 \in T A T A y=T A y$. Therefore $T A y=T A(0)=T(0)$ and, as a result, $M \cap N \subset T(0)$. Conversely, let $x \in T(0)$. Then $x \in T A(0)$ and so $x \in R(T A)$ and $T A x=T A(0)$. Hence $x \in N(T A)$. Thus $M \cap N=R(T A) \cap N(T A)=$ $T(0)$.

We have $M, N$ and $M+N=X$ are closed, then by [5, Lemma 3.1(i)], $P=T A$ is a continuous multivalued projector. On the other hand, we have $M \cap N=T(0)$ is complemented in $X$. Then by using [5, Lemma 3.1 (ii)], we deduce that $R(T)$ is complemented in $X$. Therefore $T \in \phi_{l}(X)$.

Lemma 4.3. If $T$ is an injective everywhere defined linear relation and $S$ be a bounded operator such that $S T \subset T S$, then, for all $n \in \mathbb{N}$,

$$
T^{-1}(T+S)^{-n}(0)=(T+S)^{-n}(0) \subseteq T(X) .
$$

Proof. We have $(T+S)^{-n}(0)=(T+S)^{-n} T^{-1}(0)=\left(T(T+S)^{n}\right)^{-1}(0)$. By using $\left[9\right.$, Proposition 3 (iii)] we deduce that $(T+S)^{-n}(0)=\left((T+S)^{n} T\right)^{-1}(0)=$ $T^{-1}(T+S)^{-n}(0)$. Then $T(T+S)^{-n}(0)=(T+S)^{-n}(0)+T(0)$. Hence $(T+S)^{-n}(0) \subset T(T+S)^{-n}(0) \subset T(X)$.

Proposition 4.2. Let $T$ be a bounded closed bounded below linear relation and $S$ be a compact operator such that $S T \subset T S$. Then $\operatorname{asc}(T+S)<\infty$.

Proof. We have $T$ is injective then by Lemma 4.3, we deduce that

$$
T^{-1}(T+S)^{-n}(0)=(T+S)^{-n}(0) \subseteq T(X) .
$$

If $k>0$ be such that $\|x\| \leq k\|T x\|$ for each $x \in X$, then

$$
\operatorname{dis}\left(x,(T+S)^{-n}(0)\right) \leq k \operatorname{dis}\left(Q_{T+S} T x, Q_{T+S}(T+S)^{-n}(0)\right) .
$$

Indeed; if $x \in X$ and $y_{n} \in(T+S)^{-n}(0)$ are arbitrary, then by (4.1), there exists $z_{n} \in(T+S)^{-n}(0)$ for which $y_{n} \in T z_{n},\left(T z_{n}=y_{n}+T(0)=y_{n}+\overline{T(0)}\right)$;

$$
\begin{aligned}
\operatorname{dis}\left(x,(T+S)^{-n}(0)\right) & \leq\left\|x-z_{n}\right\| \leq k\left\|T\left(x-z_{n}\right)\right\| \\
& \leq k\left\|Q_{T} T\left(x-z_{n}\right)\right\| \leq k\left\|Q_{T+S} T x-Q_{T+S} y_{n}\right\| .
\end{aligned}
$$


We deduce that $\operatorname{dis}\left(x,(T+S)^{-n}(0)\right) \leq k \operatorname{dis}\left(Q_{T+S} T x, Q_{T+S}(T+S)^{-n}(0)\right)$.

Assume that $\operatorname{asc}(T+S)=\infty$, then there exists $\left(x_{n}\right) \subset X$ such that $\left\|x_{n}\right\|=1 ; x_{n} \in(T+S)^{-n-1}(0)$ and $\operatorname{dis}\left(x_{n},(T+S)^{-n}(0)\right) \geq \frac{1}{2}$. It follows that if $n$ and $m \geq n+1$ are arbitrary, then

$$
k\left\|Q_{T+S} S x_{m}-Q_{T+S} S x_{n}\right\| \geq k\left\|Q_{T+S} S x_{n}-Q_{T+S}(T+S) x_{m}+Q_{T+S} T x_{m}\right\| .
$$

We have $x_{n} \in(T+S)^{-n-1}(0)$, then $0 \in(T+S)^{n+1}\left(x_{n}\right)$. Hence, using [9, Proposition 3], we get $0=S(0) \in S(T+S)^{n+1}\left(x_{n}\right) \subset(T+S)^{n+1}\left(S\left(x_{n}\right)\right)$. Therefore $S\left(x_{n}\right) \in(T+S)^{-n-1}(0)$. Thus,

$$
Q_{T+S} S\left(x_{n}\right) \in Q_{T+S}(T+S)^{-n-1}(0) \subset Q_{T+S}(T+S)^{-m}(0) .
$$

Now, we have $x_{m} \in(T+S)^{-m-1}(0)$, then

$$
(T+S)\left(x_{m}\right) \subset(T+S)(T+S)^{-m-1}(0)=(T+S)^{-m}(0)+(T+S)(0) .
$$

Hence $Q_{T+S}(T+S)\left(x_{m}\right) \in Q_{T+S}(T+S)^{-m}(0)$. Therefore

$$
\begin{aligned}
k\left\|Q_{T+S} S x_{m}-Q_{T+S} S x_{n}\right\| & \geq k \operatorname{dis}\left(Q_{T+S} T x_{m}, Q_{T+S}(T+S)^{-m}(0)\right) \\
& \geq \operatorname{dis}\left(x_{m},(T+S)^{-m}(0)\right) \geq \frac{1}{2}
\end{aligned}
$$

Which contradicts the compactness of the operator $Q_{T+S} S$.

Definition 4.2. We say that a relation $T \in C R(X)$ is almost left invertible if there exists $\delta>0$ such that for all $0<|\lambda|<\delta$ we have $T-\lambda I$ is left invertible.

The following theorem is our first main result of this section where we give several sufficient and necessary conditions for a closed bounded linear relation to be left-Browder.

ThEOREM 4.1. Let $T$ be a bounded closed linear relation such that $\rho(T) \neq$ $\emptyset$ and $T(0)$ is complemented in $X$. Then the following properties are equivalent:

(i) $T \in B_{l}(X)$.

(ii) $T \in \phi_{l}(X)$ and there exists $\epsilon>0$ such that for all $S \in \operatorname{comm}_{\epsilon}^{-1}(T)$, we have $T-S$ is bounded below.

(iii) $T \in \phi_{l}(X)$ and almost bounded below. 
(iv) There exists a bounded operator projector $P$, such that $T P-P T=$ $T-T, \operatorname{dim} R(P)<\infty, T$ is completely reduced by the pair $(N(P), R(P))$ with $T_{N(P)}$ is regular left invertible linear relation in $N(P)$ and $T_{R(P)}$ is a bounded nilpotent operator in $R(P)$.

(v) There exists a bounded operator projector $P$, such that $T P-P T=$ $T-T, \operatorname{dim} R(P)<\infty,(T P)^{d}=T(0)$ for some $d \in \mathbb{N}$ and $T+P$ is left invertible linear relation.

(vi) There exists a compact operator $B$ satisfying $T B-B T=T-T$ and $T-B$ is left invertible.

Proof. (i) $\Rightarrow$ (ii): An immediate consequence of Theorem 3.1.

(ii) $\Rightarrow$ (iii) : obvious.

(iii) $\Rightarrow$ (iv) : From Lemma 2.2 it follows that there exist two closed subspaces $M$ and $N$ of $X$ such that $X=M \oplus N$ with $\operatorname{dim} N<\infty ; T=T_{M} \oplus T_{N}$, such that $T_{M}$ is regular left-Fredholm linear relation in $M$ and $T_{N}$ is bounded nilpotent operator in $N$. Let $P$ be the projector such that $R(P)=N$ and $N(P)=M$. We claim that $T P-P T=T-T$. Indeed, let $x \in X$. Then there exist $x_{1} \in M$ and $x_{2} \in N$ such that $x=x_{1}+x_{2}$. Hence

$$
\begin{aligned}
(T P-P T) x & =T P x-P T x=T\left(x_{2}\right)-P\left(T_{M}\left(x_{1}\right)+T_{N}\left(x_{2}\right)\right) \\
& =T_{M}(0)+T_{N}\left(x_{2}\right)-T_{N}\left(x_{2}\right)=T_{M}(0)=T(0) .
\end{aligned}
$$

Therefore $T P-P T=T-T$.

We have $T_{R(P)}=T_{N}$ and $T_{N(P)}=T_{M}$. Evidently $T_{R(P)}$ is a bounded nilpotent operator. We claim now that $T_{N(P)}$ is a regular left invertible linear relation. Indeed, we have $T_{M}=T_{N(P)}$ is regular and left Fredholm linear relation in $N(P)$. So $R\left(T_{N(P)}\right)$ is complemented in $N(P)$. On another hand $T(0)=T_{N(P)}(0)$ is complemented in $X$. So there exists a closed subspace $F$ of $X$ such that $T(0) \oplus F=X$. Hence $(T(0) \oplus F) \cap N(P)=N(P)$. Since $T(0) \subset N(P)$, then $T(0) \oplus F \cap N(P)=N(P)$. Therefore $T(0)=T_{N(P)}(0)$ is complemented in $N(P)$.

We have $T$ is almost bounded below, then there exists $\delta>0$ such that for all $0<|\lambda|<\delta$ there exists $k_{\lambda}>0$ such that for all $x \in X ;\|x\| \leq$ $k_{\lambda}\|(T-\lambda I) x\|$. So for all $x \in N(P)$;

$$
\begin{aligned}
\|x\| & \leq k_{\lambda}\|(T-\lambda I) x\|=k_{\lambda}\left\|T_{N(P)} x-\lambda x+T_{R(P)}(0)-\lambda 0\right\| \\
& =\left\|\left(T_{N(P)}-\lambda I_{N(P)}\right) x\right\| .
\end{aligned}
$$


Therefore $T_{N(P)}$ is almost bounded below in $N(P)$. By using Theorem 2.1, we deduce that $T_{N(P)}$ is bounded below. Using Lemma 4.1, we get $T_{N(P)}$ is left invertible.

(iv) $\Rightarrow(\mathrm{v}): \quad$ Let $P$ be the projector in (iv) and $x=u+v$ such that $u \in N(P)$ and $v \in R(P)$. We have $T_{R(P)}$ is a nilpotent operator. Then there exists $d \in \mathbb{N}$ such that $T_{R(P)}^{d}=0$. Hence:

$$
\begin{aligned}
(T P)^{d} x & =\underbrace{(T P)(T P) \ldots(T P)}_{d-1} T(v) \\
& =\underbrace{(T P)(T P) \ldots(T P)}_{d-1}\left(T_{N(P)}(0)+T_{R(P)}(v)\right) .
\end{aligned}
$$

And by iteration, we get

$$
(T P)^{d} x=T_{R(P)}^{d}(v)+T(0)=T(0) .
$$

Now, if we show that $T+P$ is bounded below and $R(T+P)$ is complemented, then we can use Lemma 4.1 to deduce that $T+P$ is left invertible. For that, since $T$ is closed and $P$ is a bounded linear operator, then $T+P$ is closed.On another hand we have $T_{R(P)}$ is a bounded nilpotent operator, so $T_{R(P)}+I$ is invertible, and hence $N(T+P)=N\left(T_{N(P)}\right) \oplus N\left(T_{R(P)}+I\right)=\{0\}$ and $R(T+P)=R\left(T_{N(P)}\right) \oplus R\left(T_{R(P)}+I\right)=R\left(T_{N(P)}\right) \oplus R(P)$ which is closed. Therefore, $T+P$ is injective with closed range. Then by the closed graph theorem and Lemma 2.7 we get $T+P$ is bounded below. Now, by Proposition 4.1, $T_{N(P)}$ is $\phi_{l}(N(P))$. Then $R\left(T_{N(P)}\right)$ is complemented in $N(P)$. So there exists a closed subspace $F_{1}$ such that $R\left(T_{N(P)}\right) \oplus F_{1}=N(P)$. Therefore $R(T+P)+F_{1}=X$. Let $x \in R(T+P) \cap F_{1}$. Then $x=x_{R\left(T_{N(P)}\right)}+x_{R(P)}$ and $x=x_{F_{1}}$. So, $x_{R(P)}=x_{F_{1}}-x_{R\left(T_{N(P)}\right)}$. By according to $x_{R(P)} \in R(P)$, $x_{F_{1}}-x_{R\left(T_{N(P)}\right)} \in N(P)$ and $R\left(T_{N(P)}\right) \cap F_{1}=\{0\}$, we may deduce that $x_{R(P)}=x_{F_{1}}=x_{R\left(T_{N(P)}\right)}=0$. Therefore $x=0$. So $R(T+P) \oplus F_{1}=X$. Hence $R(T+P)$ is complemented in $X$.

$(\mathrm{v}) \Rightarrow(\mathrm{vi})$ : As, $P$ is a bounded operator with finite rank, then $P$ is compact. So, just take $B=-P$, we deduce the desired result.

(vi) $\Rightarrow$ (i) : Let $B$ be a compact operator satisfying $T B-B T=T-T$ and $T-B$ is left invertible. By Proposition 4.1, we deduce that $T-B \in \phi_{l}(X)$.

By using [2, Theorem 11] we infer that $T \in \phi_{l}(X)$. We claim now that $\operatorname{asc}(T)<+\infty$. Indeed, let $y \in B T x$. We have $T B x-B T x=T(0)$, then there exist $z \in T B x$ and $\alpha \in T(0)$ such that $y-z=\alpha$. Therefore $y \in T B x+T(0)=$ 
$T B x$. So $G(B T) \subset G(T B)$. Then

$$
B(T-B) \subset B T-B B \subset T B-B B=(T-B) B .
$$

By using Lemma 4.2 and Proposition 4.2 we deduce that $\operatorname{asc}(T)<\infty$ and, as a result, $T \in B_{l}(X)$.

4.2. Characterization of Right-Browder linear Relation. We begin by introducing the new concept of right invertible linear relation and giving some of its properties.

Definition 4.3. Let $T \in \mathcal{L R}(X)$. We say that $T$ is right invertible, if there exists a bounded operator $B$ such that $T B=I+T(0)$ and $R(B) \subset D(T)$. In this case we say that $B$ is a right inverse of $T$.

Proposition 4.3. Let $T \in \phi_{r}(X)$ be such that $T$ is bounded and onto. Then $T$ is right invertible.

Proof. We have $T$ is onto and closed, then $T$ is open. Hence $T^{-1}$ is continuous. Since $T \in \phi_{r}(X)$, then $N(T)$ is complemented. Hence there exists a continuous projector $P$ such that $R(P)=N(T)$. Let $P_{1}=I-P$ and $A=P_{1} T^{-1}$. Then, $A$ is a continuous selection of $T^{-1}$. Hence $T A x=x+T(0)$ for all $x \in X$ and $R(A) \subset R\left(T^{-1}\right)=D(T)$. Therefore $T$ is right invertible.

Remark 4.1. Let $T \in C R(X)$ be everywhere defined. If $T$ is right invertible and $T(0)$ is complemented, then there exists a bounded operator $B$ such that $T B=I+T(0)$ and $T(0)=N(B)$.

Proof. We have $T$ is right invertible, then there exists a bounded operator $A$ such that $T A=I+T(0)$. Using that $T(0)$ is complemented we deduce that there exists a closed subspace $G \subset X$ such that $T(0) \oplus G=X$. Let $B=A P_{G}$, where $P_{G}$ is the projector onto $G$ with kernel $T(0)$. Hence for all $x \in X$, we have

$$
\begin{aligned}
T B x & =T A P_{G} x=P_{G} x+T(0) \\
& =x_{G}+T(0)=x_{G}+x_{T(0)}+T(0)=x+T(0) .
\end{aligned}
$$

Therefore $T B=I+T(0)$ and $N(B) \subset T(0)$. Now, let $x \in T(0)$. Then $B x=A P_{G} x=A(0)=0$. Hence $T(0) \subset N(B)$. Therefore $T(0)=N(B)$. 
Proposition 4.4. Let $T \in C R(X)$ be everywhere defined. If $T$ is right invertible with $T(0)$ is complemented, then $T \in \phi_{r}(X)$.

Proof. Let $A$ be a right inverse of $T$ and $x \in X$. Then $T A x=x+T(0)$. Hence $x \in T A(x) \subset R(T A) \subset R(T)$. Therefore $T$ is onto and, as a result, $T \in \phi_{-}(X)$.

Now, by Remark 4.1 we deduce that there exists a bounded operator $B$ such that $T B=I+T(0)$ and $T(0)=N(B)$. Let $x \in X=D(T)$. Then there exists $y \in X$ such that $x \in T^{-1}(y)$. We have $T B y=y+T(0)$, then $T^{-1} y=B y+T^{-1}(0)$. Hence $x \in R(B)+N(T)$.

Let $x \in R(B) \cap N(T)$. Then $0 \in T x$ and there exists $y \in X$ such that $x \in B y$. Hence $0 \in T B y=y+T(0)$. Therefore $y \in T(0)$. Since $N(B)=T(0)$ we deduce that $x=0$ and so, $X=R(B) \oplus N(T)$.

Let $S:(X / N(B)) \oplus N(T) \rightarrow X$ defined by $S(\bar{x}, y)=B x+y$. We have $R(S)=R(B)+N(T)=X$, then $S$ is onto. Let $\bar{x} \in X / N(B)$ and $y \in N(T)$ be such that $S(\bar{x}, y)=B x+y=0$. Then $B x=0$ and $y=0$, hence $\bar{x}=0$ and $y=0$. Therefore $S$ is injective. We have $S$ is bijective and continuous, then $S^{-1}$ is continuous. Since $(X / N(B)) \oplus\{0\}$ is closed and $S((X / N(B)) \oplus\{0\})=$ $R(B)$, then we deduce that $R(B)$ is closed. Hence $N(T)$ is complemented and $T \in \phi_{r}(X)$.

DeFinition 4.4. We say that a relation $T \in \mathcal{L R}(X)$ is almost right invertible if there exist $\delta>0$ such that for all $0<|\lambda|<\delta$ we have $T-\lambda I$ is right invertible.

We finish this section by giving a characterization of right Browder linear relations.

Theorem 4.2. Let $X$ be a Banach space and $T \in C R(X)$ be such that $D(T)=X, T(0)$ is complemented and $\rho(T) \neq \emptyset$. Then the following properties are equivalent:

(i) $T \in B_{r}(X)$.

(ii) $T \in \phi_{r}(X)$ and there exists $\epsilon>0$ such that for all $S \in \operatorname{comm}_{\epsilon}^{-1}(T)$, we have $T-S$ is onto.

(iii) $T \in \phi_{r}(X)$, and almost onto.

(iv) There exists a bounded projector operator $P$, such that $T P-P T=T-$ $T, \operatorname{dim} R(P)<\infty, T$ is completely reduced by the pair $(N(P), R(P))$, with $T_{N(P)}$ is a regular right invertible linear relation in $N(P)$ and $T_{R(P)}$ is a bounded nilpotent operator in $R(P)$. 
(v) There exists a bounded projector operator $P$, such that $T P-P T=$ $T-T, \operatorname{dim} R(P)<\infty,(T P)^{d}=T(0)$ for some $d \in \mathbb{N}$, and $T+P$ is right invertible.

(vi) There exists a compact operator $B$ satisfying $T B-B T=T-T$ and $T-B$ is right invertible.

Proof. (i) $\Rightarrow$ (ii) : An immediate consequence of Theorem 3.2.

(ii) $\Rightarrow$ (iii) : obvious.

(iii) $\Rightarrow$ (iv): If $T \in \phi_{r}(X)$, then by Lemma 2.2 and as in the proof of Theorem 4.1 there exists a projector $P=P^{2}$, such that $T P-P T=T-T$, $T_{R(P)}$ is a bounded nilpotent operator and $T_{N(P)}$ is a regular right-Fredholm linear relation. We claim now that $T_{N(P)}$ is a bounded regular right invertible linear relation.

Indeed, we have $T$ is almost onto, then there exists $\delta>0$ such that for all $0<|\lambda|<\delta, T-\lambda$ is onto. Then, $N(P) \cap R(T-\lambda I)=N(P)$. Let $y \in$ $N(P) \cap R(T-\lambda I)=N(P)$. Then, there exists $x \in X$ such that $y \in T x-\lambda x$. Therefore there exist $x_{R(P)} \in R(P)$ and $x_{N(P)} \in N(P)$ such that

$$
y \in T_{R(P)} x_{R(P)}-\lambda x_{R(P)}+T_{N(P)} x_{N(P)}-\lambda x_{N(P)} .
$$

Then,

$$
-T_{R(P)} x_{R(P)}+\lambda x_{R(P)} \in-y+T_{N(P)} x_{N(P)}-\lambda x_{N(P)} .
$$

By using $-T_{R(P)} x_{R(P)}+\lambda x_{R(P)} \in R(P)$ and $-y+T_{N(P)} x_{N(P)}-\lambda x_{N(P)} \in$ $N(P)$ we deduce that $-T_{R(P)} x_{R(P)}+\lambda x_{R(P)}=0$. Then, $y \in R\left(T_{N(P)}-\right.$ $\left.\lambda I_{N(P)}\right)$. Therefore, $T_{N(P)}-\lambda I_{N(P)}$ is onto. So $T_{N(P)}$ is almost onto. By using $T_{N(P)}$ is regular and by Theorem 2.2 , we deduce that $T_{N(P)}$ is onto. Finally, according to $T_{N(P)} \in \phi_{r}(N(P))$ and Proposition 4.3, we infer that $T_{N(P)}$ is right invertible.

(iv) $\Rightarrow(\mathrm{v})$ : Suppose that there exists a projector $P$, such that $T P-P T=$ $T-T, T_{R(P)}$ is a nilpotent operator of degree $d$ and $T_{N(P)}$ is onto. As in the proof of Theorem 4.1 we get that $(T P)^{d}=T(0)$. From

$$
R(T+P)=R\left(T_{N(P)}\right) \oplus R\left(T_{R(P)}+I\right)=N(P) \oplus R(P)=X,
$$

we see that $T+P$ is onto. Now, we claim that $N(T+P)$ is complemented. Indeed, first we note that $N(T+P)=N\left(T_{N(P)}\right)$. On another hand, $N\left(T_{N(P)}\right)$ is complemented in $N(P)$, then there exists a closed subspace $F$ such that $N\left(T_{N(P)}\right) \oplus F=N(P)$. Hence $N\left(T_{N(P)}\right)+F+R(P)=X$. Let $x \in$ $\left(N\left(T_{N(P)}\right)+R(P)\right) \cap F$. Then $x=x_{N\left(T_{N(P)}\right)}+x_{R(P)}$ and $x=x_{F}$. Hence 
$x_{N\left(T_{N(P)}\right)}+x_{R(P)}=x_{F}$. So, $x_{R(P)}=x_{F}-x_{N\left(T_{N(P)}\right)}$. By according to $x_{R(P)} \in R(P)$ and $x_{F}-x_{N\left(T_{N(P)}\right)} \in N(P)$, we may deduce that $x_{R(P)}=0$ and $x_{F}=x_{N\left(T_{N(P)}\right)}$. Therefore $x_{F}=0$ and $x_{N\left(T_{N(P)}\right)}=0$. Thus $x=0$. So $N(T+P)$ is complemented in $X$. Then $T+P \in \phi_{r}(X)$ such that $T+P$ is onto. By using Proposition 4.3 we deduce that $T+P$ is right invertible.

$(\mathrm{v}) \Rightarrow(\mathrm{vi})$ : As, $P$ is a bounded operator with finite rank, then $P$ is compact. So, just take $K=-P$, we deduce that there exists a compact operator $K$ satisfying $T K-K T=T-T$ and $T-K$ is right invertible.

(vi) $\Rightarrow$ (i): We have $T-K$ is right invertible, then by Proposition 4.4 we deduce the desired result.

We have $(T-K)(0)=T(0)$ is closed, $K(T-K) \subset(T-K) K$ and $T-K$ is onto, then by [9, Proposition 14] we deduce that $d(T)<\infty$. Therefore $T \in B_{r}(X)$.

\section{REFERENCES}

[1] T. Alvarez, On Regular Linear Relations, Acta Math. Sin. (Engl. Ser.) 28 (1) (2012), 183-194.

[2] T. Alvarez, On the perturbation of semi-Fredholm relations with complemented ranges and null spaces, Acta Math. Sin. (Engl. Ser.) 26 (8) (2010), 1545-1554. Published online: July 15, 2010.

[3] T. Alvarez, M. Benharrat, Relationship Between the Kato Spectrum and the Goldberg Spectrum of a Linear Relation, Mediterr. J. Math. 13 (1) (2016), $365-378$.

[4] T. Alvarez, D. Wilcox, Perturbation theory of multivalued Atkinson operators in normed spaces, Bull. Austral. Math. Soc. 76 (2) (2007), 195-204.

[5] T. Alvarez, Y. Chamkha, M. Mnif, Left- and right-Atkinson linear relation matrices, Mediterr. J. Math. 13 (4) (2016), 2039-2059.

[6] T. Alvarez, F. Fakhfakh, M. Mnif, Coperturbation function and lower semi-Browder multivalued linear operators, Linear Multilinear Algebra 61 (4)

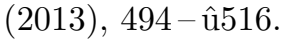

[7] T. Alvarez, F. Fakhfakh, M. Mnif, Left-right Fredholm and left-right Browder linear relations, Filomat 31 (2) (2017), 255-271.

[8] R.W. Cross, "Multivalued Linear Operators", Monographs and Textbooks in Pure and Applied Mathematics, 213. Marcel Dekker, Inc., New York, 1998.

[9] F. Fakhfakh, M. Mnif, Perturbation theory of lower semi-Browder multivalued linear operators, Publ. Math. Debrecen 78 (3-4) (2011), 595-606.

[10] V. MÜLLER, "Spectral Theory of Linear Operators and Spectral Systems in Banach Algebras", Second edition, Operator Theory: Advances and Applications, 139. Birkhäuser Verlag, Basel, 2007. 
[11] C. Snezana Zivkovic-Zlatanovic, Dragan S. Djordjevic, Robin E. HARTe, On left and right Browder operators, J. Korean Math. Soc. 48 (5) (2011), 1053-1063.

[12] D. Wilcox, Essential spectra of linear relations, Linear Algebra Appl. 462 (2014), $110-125$. 